\title{
A Modified Scheme of Triplectic Quantization
}

\author{
B. Geyer \\ Naturwissenschaftlich-Theoretisches Zentrum und \\ Institut für Theoretische Physik, Universität Leipzig, \\ Augustusplatz 10/11, 04109 Leipzig, Germany \\ D.M. Gitman \\ Instituto de Fisica, Universidade de São Paulo \\ Caixa Postal 66318-CEP, 05315-970-São Paulo, S.P., Brazil \\ P.M. Lavrov \\ Tomsk State Pedagogical University, 634041 Tomsk, Russia
}

\begin{abstract}
A modified version of triplectic quantization, first introduced by Batalin and Marnelius, is proposed which makes use of two independent master equations, one for the action and one for the gauge functional such that the initial classical action also obeys that master equation.
\end{abstract}

\section{Introduction}

Lagrangian quantization (LQ) remains one of the most attractive approaches to quantize gauge theories. Its main advantage is a direct construction of the quantum effective action avoiding the long detour of the Hamiltonian approach through canonical quantization with subsequent integration over momenta in the path integral, and producing directly the vacuum expectation value of the S-matrix in the presence of external sources (see, for example, [回). The main difficulty faced by the implementation of the LQ program lies in the complicated structure of the gauge symmetries corresponding to the initial classical action of a theory, as well as in the ambiguities existing in the choice of possible gauges. It is well known that gauge theories with closed algebra of linearly independent generators of gauge transformations - when considered in the class of certain admissible (although restricted) gauges - permit the quantum effective action $S_{\text {eff }}$ to be constructed directly by means of the Faddeev-Popov (FP) rules [2]. The overwhelming majority of physically interesting theories - such as Yang-Mills theories, theory of gravity, etc - belong to the realm of the application of this quantization method. According to the FP rules, the action $S_{\text {eff }}$ depends on an enlarged set of classical fields $\phi$, composed, apart from the basic classical fields $A$, also by the sets of auxiliary fields $B$ (Nakanishi-Lautrup fields) related to the gauge-fixing, as well as by the sets of FP ghost and anti-ghost fields $C, \bar{C}$ : altogether they are given by $\phi=(A, B, C, \bar{C})$. The action $S_{\text {eff }}$ constructed according to the $\mathrm{FP}$ rules is invariant under the (global) nilpotent BRST transformations [3, 4]. Assigning to each field $\phi$ the corresponding BRST-source, or anti-field, $\phi^{*}$, one observes that the BRST symmetry results in the nonlinear Zinn-Justin equation [5] determining the quantum action $S$ which ensures complete description of $S_{\text {eff }}$.

Attempts to go beyond the scope of the above mentioned restrictions on the structure of gauge symmetries of the initial classical action - as well as beyond the restrictions on the 
above mentioned classes of gauge conditions - have resulted in a number of new schemes of LQ, based of the so-called master equations (analogous to the Zinn-Justin equation). Their solutions determine the quantum action $S$, which satisfies a set of natural boundary conditions related to the initial classical action. One of the first schemes of this kind was the Batalin-Vilkovisky (BV) method [6, 7], which allows to consider gauge theories with open gauge algebra. In the case of irreducible theories [6], corresponding to a linearly independent set of generators, the configuration space $\phi$ of the BV method coincides with that of the FP approach, whereas in the case of reducible theories [7] one has to introduce additional (sometimes infinite) sets of auxiliary and ghost fields. Afterwards the so-called $S p(2)$ symmetric scheme has been developed [8, 9, 10, in which the ghost and anti-ghost fields are treated on equal footing (in contrast to the BV method, where the anti-ghost fields appear only at the stage of gauge-fixing), and in which the (in)finite towers of auxiliary and ghost fields are classified in terms of irreducible representations of the $S p(2)$ group. In fact, such a quantization scheme realizes a combined BRST anti-BRST symmetry principle (the latter symmetry was first discovered in [11, 12] for Yang-Mills theories in the framework of the FP method). Within the $S p(2)$ scheme, the quantum action $S$ depends on an extended set of field variables, which includes, apart from the variables $\phi$ also three sets of anti-fields, namely, $S p(2)$-doublets $\phi_{a}^{*}, a=1,2$ (sources of BRST and anti-BRST transforms) and $S p(2)$-scalars $\phi$ (sources of mixed transforms).

In a recent work 13 Batalin and Marnelius presented a new possible generalization of the $S p(2)$ method - related to an additional extension of the configuration space of the quantum action $S$ - and aimed at providing equal treatment for all anti-fields of the $S p(2)$ approach. In particular, it is suggested to consider the fields $\pi^{a}$ (which parametrize gauges in the path integral of the $S p(2)$ method) as anticanonically conjugated to the anti-fields $\bar{\phi}$, with the corresponding redefinition of the extended anti-brackets. Thus, along with the $S p(2)$-singlet $\bar{\phi}$, the theory also contains an $S p(2)$-doublet $\pi^{a}$, which thus accounts for the fact that the corresponding scheme is referred to as triplectic quantization (TQ). An essential original point of the TQ scheme consists in dividing the entire task of constructing the quantum effective action $S_{\text {eff }}$ into the following two steps: first, the construction of the quantum action $S$, and second the construction of the corresponding gauge-fixing functional. Either problem is solved by means of an appropriate master equation.

Despite considering these new ideas as very promising, as to their concrete realization we propose a different, modified scheme of the TQ, which - especially from some geometrical viewpoint - changes the meaning of the latter. Namely, remaining in the same configuration space of fields, and accepting the idea of a separate treatment of the two above mentioned actions, we propose to change both systems of master equations by using a new set of two $S p(2)$-doublets of generating operators: $V^{a}$ and $U^{a}$. Such a modification is inspired by our experience in the superfield formulation of the $S p(2)$ method [14], in which the above operators acquire the geometrical interpretation of the generators of (super)transformations in a superspace spanned by fields and anti-fields. In this approach, the first master equation, determining the quantum action $S$, is defined by means of the operators $V^{a}$, whereas the other master equation, determining the gauge fixing functional $X$, is defined by means of the operators $U^{a}$. As in the original TQ scheme, we may expect that the generating functional of Green's functions does not depend on the choice of gauge. It is important to emphasize that within the modified TQ scheme the entire information contained in the initial classical action of the theory is conveyed to the quantum effective action via the corresponding boundary conditions. At the same time, the classical action obeys the first modified master equation in complete analogy with all previously known schemes of LQ. The original TQ scheme gives no explicit relation to the initial classical action. If one assumes that such a classical action occurs, as usual, in the boundary condition to the solution of the master equation (with vanishing auxiliary fields 
and quantum corrections), then this classical action does not obey the master equation.

The purpose of this paper is to elaborate a complete description of TQ scheme - within the framework of the above mentioned modifications - revealing, at the same time, the points which make such a description similar to, or different from, the original TQ scheme.

\section{Main Definitions}

Let us denote by $\phi^{A}, \varepsilon\left(\phi^{A}\right) \equiv \varepsilon_{A}$, the complete set of fields which span the configuration space corresponding to a certain initial gauge theory. The explicit structure of the fields $\phi^{A}$ is not essential for the purposes of the following treatment (for details, see the original papers [6, 7]). According to refs. [8, 13] we further introduce the set of antifields $\phi_{A a}^{*}$, $\varepsilon\left(\phi_{A a}^{*}\right)=\varepsilon_{A}+1 ; \bar{\phi}_{A}, \varepsilon\left(\bar{\phi}_{A}\right)=\varepsilon_{A}$, accompanied [13] by the set of fields $\pi^{A a}, \varepsilon\left(\pi^{A a}\right)=\varepsilon_{A}+1$. In the entire space of variables $\phi^{A}, \phi_{A a}^{*}, \pi^{A a}, \phi_{A}$ we define extended antibrackets, given, for any two functionals $F, G$, by the rule

$$
(F, G)^{a}=\frac{\delta F}{\delta \phi^{A}} \frac{\delta G}{\delta \phi_{A a}^{*}}+\varepsilon^{a b} \frac{\delta F}{\delta \pi^{A b}} \frac{\delta G}{\delta \bar{\phi}_{A}}-(F \leftrightarrow G)(-1)^{(\varepsilon(F)+1)(\varepsilon(G)+1)}
$$

where $\varepsilon^{a b}$ is the constant antisymmetric second-rank tensor, $\varepsilon^{12}=1$. Eq. (1) coincides with the definition of the extended antibrackets given in the method of triplectic quantization [13], as well as with the definition of the extended antibrackets given in the method of superfield quantization [14]. Speaking of the algebraic properties of the extended antibrackets, we will only mention the generalized Jacobi identities

$$
\left((F, G)^{\{a}, H\right)^{b\}}(-1)^{(\varepsilon(F)+1)(\varepsilon(H)+1)}+\operatorname{cycle}(F G H) \equiv 0 .
$$

Here and elsewhere the curly brackets stand for symmetrization over $a, b$.

In solving the functional equations determining the effective action we make use of the operators $\Delta^{a}, V^{a}$ and $U^{a}$

$$
\begin{aligned}
\Delta^{a} & =(-1)^{\varepsilon_{A}} \frac{\delta_{l}}{\delta \phi^{A}} \frac{\delta}{\delta \phi_{A a}^{*}}+(-1)^{\varepsilon_{A}+1} \varepsilon^{a b} \frac{\delta_{l}}{\delta \pi^{A b}} \frac{\delta}{\delta \bar{\phi}_{A}}, \\
V^{a} & =\varepsilon^{a b} \phi_{A b}^{*} \frac{\delta}{\delta \bar{\phi}_{A}} \\
U^{a} & =(-1)^{\varepsilon_{A}+1} \pi^{A a} \frac{\delta_{l}}{\delta \phi^{A}} .
\end{aligned}
$$

Notice that the operators $\Delta^{a}$ have already appeared both within the scheme of triplectic quantization |13] and, virtually, within the scheme of superfield quantization [14]. Even though the operators $V^{a}$ in eq. (4) differ from the corresponding operators of the triplectic quantization [13], they coincide, at the same time, with the operators applied in the framework of the $S p(2)$ method [8]. The use of the operators $U^{a}$ in eq. (5) (an analog of these operators has been introduced in the method of superfield quantization) exhibits an essentially new feature as compared to both the $S p(2)$ method and the TQ [13].

One easily establishes the following algebra of the operators (3)-(5):

$$
\begin{aligned}
& \Delta^{\{a} \Delta^{b\}}=0, \\
& V^{\{a} V^{b\}}=0, \\
& \Delta^{\{a} V^{b\}}+V^{\{a} \Delta^{b\}}=0, \\
& U^{\{a} U^{b\}}=0,
\end{aligned}
$$




$$
\begin{aligned}
& \Delta^{\{a} U^{b\}}+U^{\{a} \Delta^{b\}}=0, \\
& V^{a} U^{b}+U^{b} V^{a}=0, \\
& \Delta^{a} V^{b}+V^{b} \Delta^{a}+\Delta^{a} U^{b}+U^{b} \Delta^{a}=0 .
\end{aligned}
$$

The action of the operators $\Delta^{a}(3)$ on the product of any two functionals $F, G$,

$$
\Delta^{a}(F \cdot G)=\left(\Delta^{a} F\right) \cdot G+F \cdot\left(\Delta^{a} G\right)(-1)^{\varepsilon(F)}+(F, G)^{a}(-1)^{\varepsilon(F)},
$$

may serve as an independent definition of the extended antibrackets (1). The action of each of the operators $\Delta^{a}, V^{a}$ and $U^{a}(3)-(5)$ on the extended antibrackets is given by the rule $\left(D^{a}=\left(\Delta^{a}, V^{a}, U^{a}\right)\right)$

$$
D^{\{a}(F, G)^{b\}}=\left(D^{\{a} F, G\right)^{b\}}-\left(F, D^{\{a} G\right)^{b\}}(-1)^{\varepsilon(F)} .
$$

Apart from $\Delta^{a}, V^{a}$, we also introduce the operators

$$
\begin{aligned}
\bar{\Delta}^{a} & \equiv \Delta^{a}+\frac{i}{\hbar} V^{a}, \\
\tilde{\Delta}^{a} & \equiv \Delta^{a}-\frac{i}{\hbar} U^{a} .
\end{aligned}
$$

From eqs. (6)-(12) it follows that the algebra of these operators has the form

$$
\begin{aligned}
& \bar{\Delta}^{\{a} \bar{\Delta}^{b\}}=0, \\
& \tilde{\Delta}^{\{a} \tilde{\Delta}^{b\}}=0, \\
& \bar{\Delta}^{\{a} \tilde{\Delta}^{b\}}+\tilde{\Delta}^{\{a} \bar{\Delta}^{b\}}=0 .
\end{aligned}
$$

\section{Generating Functional, Extended BRST Symmetry and Gauge Independence}

Let us denote by $S=S\left(\phi, \phi^{*}, \pi, \bar{\phi}\right)$ the quantum action, corresponding to the initial classical theory with the action $S_{0}$, and defined as a solution of the following master equations:

$$
\frac{1}{2}(S, S)^{a}+V^{a} S=i \hbar \Delta^{a} S
$$

with the standard boundary condition

$$
\left.S\right|_{\phi^{*}=\bar{\phi}=\hbar=0}=S_{0}
$$

Eq. (20) can be represented in the equivalent form

$$
\bar{\Delta}^{a} \exp \left\{\frac{i}{\hbar} S\right\}=0 .
$$

Let us further define the vacuum functional as the following functional integral:

$$
Z_{X}=\int d \phi d \phi^{*} d \pi d \bar{\phi} d \lambda \exp \left\{\frac{i}{\hbar}\left(S+X+\phi_{A a}^{*} \pi^{A a}\right)\right\}
$$


where $X=X\left(\phi, \phi^{*}, \pi, \bar{\phi}, \lambda\right)$ is a bosonic functional depending also on the new variables $\lambda^{A}, \varepsilon(\lambda)=\varepsilon_{A}$, which serve as gauge-fixing parameters. We require that the functional $X$ satisfies the following master equation:

$$
\frac{1}{2}(X, X)^{a}-U^{a} X=i \hbar \Delta^{a} X
$$

or, equivalently,

$$
\tilde{\Delta}^{a} \exp \left\{\frac{i}{\hbar} X\right\}=0
$$

Notice that the generating equations determining the quantum action $S$ in eq. (20) (or (22)) and the gauge-fixing functional $X$ in eq. (24) (or (25)) differ - along with the vacuum functional $Z$ in eq. (23) - from the corresponding definitions applied in the method of TQ [13.

One can easily obtain the simplest solution of eq. (24) (or eq. (25)) determining the gauge-fixing functional $X$

$$
\begin{aligned}
X & =\left(\bar{\phi}_{A}-\frac{\delta F}{\delta \phi^{A}}\right) \lambda^{A}-\frac{1}{2} \varepsilon_{a b} U^{a} U^{b} F= \\
& =\left(\bar{\phi}_{A}-\frac{\delta F}{\delta \phi^{A}}\right) \lambda^{A}-\frac{1}{2} \varepsilon_{a b} \pi^{A a} \frac{\delta^{2} F}{\delta \phi^{A} \delta \phi^{B}} \pi^{B b}
\end{aligned}
$$

where $F=F(\phi)$ is a bosonic functional depending only on the fields $\phi^{A}$. As a straightforward exercise, one makes sure that the functional $X$ in eq. (26) does satisfy eq. (24). If we further demand that the quantum action $S$ does not depend on the fields $\pi^{A}$, then the functional (26) becomes exactly the vacuum functional of the $S p(2)$ quantization scheme [8, 9.

Let us consider a number of properties inherent in the present scheme of triplectic quantization, i.e. modified according to eq. (20)-(25). In the first place, the vacuum functional (23) is invariant under the following transformations:

$$
\delta \Gamma=(\Gamma,-S+X)^{a} \mu_{a}+\mu_{a}\left(V^{a}+U^{a}\right) \Gamma,
$$

where $\mu_{a}$ is an $S p(2)$ doublet of constant anticommuting parameters, and $\Gamma$ stands for any of the variables $\phi, \phi^{*}, \pi, \bar{\phi}$. Eq. (27) defines the transformations of extended BRST symmetry, realized on the space of the variables $\phi, \phi^{*}, \pi, \bar{\phi}$. In the particular case, corresponding to the gauge-fixing boson chosen as in eq. (26), we have

$$
\begin{aligned}
\delta \phi^{A} & =-\left(\frac{\delta S}{\delta \phi_{A a}^{*}}-\pi^{A a}\right) \mu_{a}, \\
\delta \phi_{A a}^{*} & =\mu_{a}\left(\frac{\delta S}{\delta \phi^{A}}+\frac{\delta^{2} F}{\delta \phi^{A} \delta \phi^{B}} \lambda^{B}+\frac{1}{2}(-1)^{\varepsilon_{A}} \varepsilon_{b c} \pi^{B b} \frac{\delta^{3} F}{\delta \phi^{B} \delta \phi^{A} \delta \phi^{C}} \pi^{C c}\right) \\
\delta \pi^{A a} & =\varepsilon^{a b}\left(\frac{\delta S}{\delta \bar{\phi}_{A}}-\lambda^{A}\right) \mu_{b}, \\
\delta \bar{\phi}_{A} & =\mu_{a} \varepsilon^{a b}\left(\frac{\delta S}{\delta \pi^{A b}}+\phi_{A b}^{*}\right)+\mu_{a} \frac{\delta^{2} F}{\delta \phi^{A} \delta \phi^{B}} \pi^{B a} .
\end{aligned}
$$

Consider now the question of gauge dependence in the case of the vacuum functional $Z$, eq. (23). Any admissible variation $\delta X$ should satisfy the equations

$$
(X, \delta X)^{a}-U^{a} \delta X=i \hbar \Delta^{a} \delta X .
$$


It is convenient to consider an $S p(2)$ doublet of operators $\hat{S}^{a}(X)$, defined by the rule

$$
(X, F)^{a} \equiv \hat{S}^{a}(X) \cdot F,
$$

and possessing the properties

$$
\hat{S}^{\{a}(X) \hat{S}^{b\}}(X)=\hat{S}^{\{a}\left(\frac{1}{2}(X, X)^{b\}}\right),
$$

which follow from the generalized Jacobi identities (2). Eq. (32) can be, consequently, represented in the form

$$
\hat{Q}^{a}(X) \delta X=0
$$

where we have introduced an $S p(2)$ doublet of operators $\hat{Q}^{a}$, defined by the rule

$$
\hat{Q}^{a}(X)=\hat{S}^{a}(X)-i \hbar \tilde{\Delta}^{a} .
$$

With allowance for eq. (24) the operators $\hat{Q}^{a}$ form a set of nilpotent anticommuting operators, i.e.

$$
\hat{Q}^{\{a}(X) \hat{Q}^{b\}}(X)=0
$$

By virtue of eq. (37), any bosonic functional of the form

$$
\delta X=\frac{1}{2} \varepsilon_{a b} \hat{Q}^{a}(X) \hat{Q}^{b}(X) \delta Y,
$$

with an arbitrary bosonic functional $\delta Y$, is a solution of eq. (35). Moreover, by analogy with the theorems proved in ref. [10], one establishes the fact that any solution of eq. (35)vanishing when all the vari in $\delta X$ are equal to zero - has the form (38), with a certain bosonic functional $\delta Y$. In the particular case of the gauge functional $X(26)$, its variation $\delta X$ can be easily represented in the form of eq. (38), i.e.

$$
\delta X=-\frac{\delta(\delta F)}{\delta \phi^{A}} \lambda^{A}-\frac{1}{2} \varepsilon_{a b} \pi^{A a} \frac{\delta^{2}(\delta F)}{\delta \phi^{A} \delta \phi^{B}} \pi^{B b}=-\frac{1}{2} \varepsilon_{a b} \hat{Q}^{a}(X) \hat{Q}^{b}(X) \delta F
$$

with $\delta Y=-\delta F$.

Let us denote by $Z_{X} \equiv Z$ the value of the vacuum functional (23) corresponding to the gauge condition chosen as a functional $X$.

In the vacuum functional $Z_{X+\delta X}$ we first make the change of variables (27), with $\mu_{a}=\mu_{a}(\Gamma, \lambda)$, and then, accompanying it with a subsequent change of variables

$$
\delta \Gamma=\left(\Gamma, \delta Y_{a}\right)^{a}, \quad \varepsilon\left(\delta Y_{a}\right)=1,
$$

with $\delta Y_{a}=-i \hbar \mu_{a}(\Gamma, \lambda)$, we arrive at

$$
Z_{X+\delta X}=\int d \phi d \phi^{*} d \pi d \bar{\phi} d \lambda \exp \left\{\frac{i}{\hbar}\left(S+X+\delta X+\delta X_{1}+\phi_{A a}^{*} \pi^{A a}\right)\right\}
$$

In eq. (41) we have used the notation

$$
\delta X_{1}=2\left(\left(X, \delta Y_{a}\right)^{a}-U^{a} \delta Y_{a}-i \hbar \Delta^{a} \delta Y_{a}\right)=2 \hat{Q}^{a}(X) \delta Y_{a}
$$


Let us choose the functional $\delta Y_{a}$ in the form

$$
\delta Y_{a}=\frac{1}{4} \varepsilon_{a b} \hat{Q}^{b} \overline{\delta Y}, \quad \varepsilon(\overline{\delta Y})=0
$$

Then, representing $\delta X$ as in eq. (38), and identifying $\delta Y=-\overline{\delta Y}$, we find that

$$
Z_{X+\delta X}=Z_{X}
$$

i.e. the vacuum functional (and hence, by virtue of the equivalence theorem [15], also the $S$ matrix) does not depend on the choice of gauge. Note that in the particular case (39) we have $\overline{\delta Y}=\delta F$.

\section{Concluding Remarks}

The reader may profit by considering the original [13] version of TQ as compared to the modified scheme, proposed in the this paper. Thus, both versions are based on extended BRST symmetry. Both versions apply the vacuum functional and the $S$ matrix not depending on the choice of gauge, while admitting of gauges which reproduce the results of the $S p(2)$ symmetric quantization. Both versions implement the idea of separate treatment of the quantum action and the gauge-fixing functional, based each on appropriate master equations. The principal distinctions concern a different form of these equations as well as a different form of the vacuum functional. The modification of the generating equations [13] permits incorporating the information contained in the initial classical action by means of the corresponding boundary conditions. In contrast to the original version [13], the classical action provides a solution of the modified master equation. Thus, one establishes a connection with the previous schemes of LQ. In particular, one easily reveals the fact of equivalence with the $S p(2)$ quantization, by means of explicit realization of the corresponding class of boundary condition. In the original version of TQ,

however, these questions still remain open. Another distinction of the two TQ schemes is connected with the explicit structure of the corresponding master equations. Thus, the original version |13] of TQ defined the generating equations for the quantum action and the vacuum functional, using the operators

$$
V_{\mathrm{BM}}^{a}=\frac{1}{2}\left(\varepsilon^{a b} \phi_{A b}^{*} \frac{\delta}{\delta \bar{\phi}_{A}}+\pi^{A a}(-1)^{\varepsilon_{A}+1} \frac{\delta_{l}}{\delta \phi^{A}}\right)=\frac{1}{2}\left(U^{a}+V^{a}\right) .
$$

The use of the generating equations determining the quantum action with the help of the operators $V_{\mathrm{BM}}^{a}$ leads to the following characteristic feature of the triplectic quantization [13]: the classical action of the initial theory, defined as a limit of the quantum action at $\hbar \rightarrow 0$ and $\phi^{*}=\bar{\phi}=\pi=0$, does not satisfy the generating equations of the method. In turn, the proofs of the existence theorems for the generating equations in all known methods of LQ are based on the fact that the initial classical action is a solution of the corresponding master equations. Moreover, form the viewpoint of the superfield quantization [14], which applies operators $V^{a}, U^{a}$, whose component representation is

$$
\begin{aligned}
V^{a} & =\varepsilon^{a b} \phi_{A b}^{*} \frac{\delta}{\delta \bar{\phi}_{A}}-J_{A} \frac{\delta}{\delta \phi_{A a}^{*}}, \\
U^{a} & =(-1)^{\varepsilon_{A}+1} \pi^{A a} \frac{\delta_{l}}{\delta \phi^{A}}+(-1)^{\varepsilon_{A}} \varepsilon^{a b} \lambda^{A} \frac{\delta_{l}}{\delta \pi^{A b}}
\end{aligned}
$$


(with $J_{A}$ being the sources to the fields $\phi^{A}$ ), the operators (45) have no precise geometrical meaning, whereas the $V^{a}$ and $U^{a}$ in eq. (46) serve as generators of supertranslationsin superspace spanned by superfields and superantifields - along additional (Grassmann) coordinates. In turn, the operators $V^{a}(4)$ and $U^{a}(5)$, applied in this paper, can be considered as limits (at $J_{A}=0, \lambda^{A}=0$ ) of the operators (46), which possess a clear geometrical meaning. The present modified scheme of triplectic quantization enjoys every attractive feature of the quantization [13]: the theory possesses extended BRST transformations; the vacuum functional and the $S$ matrix do not depend on the choice of the gauge-fixing functional; there exists such a choice of the gauge-fixing functional and solutions of the generating equations that reproduces the results of the $S p(2)$ method.

\section{Acknowledgments}

The work of one of the authors (PML) has been supported by the Russian Foundation for Basic Research (RFBR), project 96-02-16017, as well as by grant INTAS 96-0308 and by grant RFBR-DFG 96-02-00180. PML appreciates the kind hospitality extended to him by the Center of Advanced Study (NTZ) of Leipzig University and by the Institute of Physics of the University of São Paulo. DMG thanks Brazilian Foundation CNPq and FAPESP for partial support.

\section{References}

[1] D.M. Gitman and I.V. Tyutin, Canonical quantization of fields with constraints (Nauka, Moscow, 1986); Quantization of Fields with Constraints, (Springer-Verlag, Berlin 1990)

[2] L.D. Faddeev and V.N. Popov, Phys. Lett. B25 (1967) 29

[3] C. Becchi, A. Rouet, and R. Stora, Phys. Lett. B25 (1974) 344; Comm. Math. Phys. $42(1975) 127$

[4] I.V. Tyutin, Preprint Lebedev Inst. No. 35 (1975)

[5] Zinn-Justin, Renormalization of gauge theories in Trends in elementary particle theory, Lectures notes in Physics, 37 (Springer-Verlag, Berlin 1975)

[6] I.A. Batalin and Vilkovisky, Phys. Lett. B102 (1981) 27

[7] I.A. Batalin and Vilkovisky, Phys. Rev. D28 (1983) 2567

[8] I.A. Batalin, P.M. Lavrov, and I.V. Tyutin, J. Math. Phys. 31 (1990)

[9] I.A. Batalin, P.M. Lavrov, and I.V. Tyutin, J. Math. Phys. 32 (1991)

[10] I.A. Batalin, P.M. Lavrov, and I.V. Tyutin, J. Math. Phys. 32 (1991)

[11] G. Curci and R. Ferrari, Phys. Lett. B63 (1976) 91

[12] I. Ojima, Prog. Theor. Phys. Suppl. 64 (1979) 625

[13] I.A. Batalin and R. Marnelius, Phys. Lett. B350 (1995) 44

[14] P.M. Lavrov, Phys. Lett. B366 (1995) 160

[15] R.E. Kallosh and I.V. Tyutin, Yad. Fiz. 17 (1973) 190 